\title{
LINC00238 inhibits hepatic carcinoma progression by activating TMEM106C-mediated apoptosis pathway
}

\author{
CAIHUA JIANG ${ }^{1 *}$, FENG LI $^{2 *}$, MENG YANG $^{3}$, JIANPING DUAN $^{2}$, \\ JIANMING LAI ${ }^{4}$, SHULUN SUN $^{5}$ and SHAOHUA FAN ${ }^{6}$
}

\begin{abstract}
Departments of ${ }^{1}$ Outpatients, ${ }^{2}$ Infectious Disease and ${ }^{3}$ Physical Therapy, Qingdao No. 6 People's Hospital, Qingdao, Shandong 266033; ${ }^{4}$ Qingdao University Medical College, Qingdao, Shandong 266071;

Departments of ${ }^{5}$ Liver Disease and ${ }^{6}$ Blood Purification Center,

Qingdao No. 6 People's Hospital, Qingdao, Shandong 266033, P.R. China
\end{abstract}

Received February 6, 2021; Accepted June 22, 2021

DOI: $10.3892 / \mathrm{mmr} .2021 .12397$

\begin{abstract}
The present study aimed to explore the regulatory mechanism of long intergenic non-protein coding (LINC) 00238 in hepatocellular carcinoma (HCC). LINC00238 expression in HCC tissues and cell lines was measured using reverse transcription-quantitative PCR. LncTar was used to predict the binding sites between LINC00238 and transmembrane protein 106C (TMEM106C). Survival analysis of LINC00238, TMEM106C and activating transcription factor 3 (ATF3) in patients with HCC was performed based on TCGA data. The proliferation, apoptosis, migration, and invasion of HCC cells were measured by 3-(4,5-dimethylthiazol-2-yl)-5-(3-carboxy methoxyphenyl)-2-(4-sulfophenyl)-2H-tetrazolium assay, flow cytometer, wound healing and Transwell assays, respectively. LINC00238 promoted apoptosis and inhibited proliferation, migration and invasion of HCC cells. LINC00238 was downregulated in HCC. TMEM106C was a target of LINC00238 and TMEM106C expression was negatively regulated by LINC00238. TMEM106C suppressed the apoptosis pathway and decreased the expression of caspase-7, tissue inhibitor of metalloproteinase 2, programmed cell death 4 and ATF3. Notably, ATF3 was the upstream promoter of LINC00238 and positively regulated LINC00238 expression.
\end{abstract}

Correspondence to: Dr Shulun Sun, Department of Liver Disease, Qingdao No. 6 People's Hospital, 9 Fushun Road, Shibei, Qingdao, Shandong 266033, P.R. China

E-mail: Sunshulun123@126.com

Dr Shaohua Fan, Department of Blood Purification Center, Qingdao No. 6 People's Hospital, 9 Fushun Road, Shibei, Qingdao, Shandong 266033, P.R. China

E-mail: ShaohuaFan1123@126.com

*Contributed equally

Key words: hepatocellular carcinoma, long intergenic non-protein coding RNA 00238, transmembrane protein 106C, activating transcription factor 3
In conclusion, LINC00238 inhibited HCC progression by inhibiting TMEM106 expression and activating the TMEM106C-mediated apoptosis pathway.

\section{Introduction}

Hepatocellular carcinoma (HCC) is the fourth major cause of cancer mortality worldwide and the global morbidity of the disease is increasing (1). Surgical resection is the most effective treatment method of all therapy methods; however, most patients are initially diagnosed as advanced and untreatable by surgery (2). High rates of postoperative recurrence and metastasis are also reasons for the high mortality rate of HCC (3). Although great progress in the therapy methods has been made in the last few decades, the survival rate of patients with HCC remains poor (4). Hence, the search for new effective therapeutic targets is of great significance for HCC treatment.

In recent years, an increasing numbers of studies have focused on the effect of IncRNAs on the occurrence and development of HCC (5-7). Research has shown that aberrant expression of long non-coding RNAs (lncRNAs) decelerates or accelerates the progression of HCC. For instance, a study by Liu et al (8) demonstrates that lncRNA Ftx can inhibit proliferation and metastasis of HCC and that higher lncRNA Ftx expression is associated with longer survival. Furthermore, long intergenic non-protein coding (LINC)RNA-p21 overexpression reduces the levels of epithelial-mesenchymal transition (EMT)-related proteins and decrease tumor metastasis in HCC (7). The findings of Wang et al (9) indicate that high lncRNA ATB expression promotes proliferation of HCC and is positively correlated with poorer survival of patients with HCC. Gong et al (6) found that LINC00238 is expressed at low levels in HCC samples. However, the regulatory mechanism of LINC00238 in HCC remains to be elucidated.

The transmembrane protein (TMEM) family is a class of proteins containing one or more putative transmembrane segments that span partially or completely through biological membranes (10). As a member of the TMEM family, TMEM106 is involved in several diseases, including cancer. In a study on non-small cell lung carcinoma, Liu and Zhu (11) found that TMEM106A is an anti-oncogene that inhibits the 
proliferation and invasion of lung cancer cells. Lang et al (12) note that abnormal expression of TMEM106B is associated with progression of frontotemporal lobar degeneration. In addition, Assassi et al (13) found that TMEM106C is aberrantly expressed in ankylosing spondylitis. Luo et al (14) have indicate that TMEM106C is involved in HCC development. However, remains to be elucidated whether lncRNAs are involved in the regulation of TMEM106C in HCC progression.

The present study explored the regulatory role of LINC00238 in HCC progression. The findings suggested that LINC00238 expression was decreased in HCC tissues and cell lines and the low LINC00238 expression was associated with the poor prognosis of patients with HCC. LINC00238 inhibited HCC progression by regulating the TMEM106C-mediated apoptosis signaling pathway. Furthermore, ATF3, the gene enriched in the apoptosis pathway, could regulate LINC00238 expression, consequently preventing $\mathrm{HCC}$ development.

\section{Materials and methods}

Bioinformatics analysis. The online platform Gene Expression Profiling Interactive Analysis (GEPIA) (15) was used to analyze the differentially expressed IncRNAs in HCC. A total of $369 \mathrm{HCC}$ cases and 160 normal were downloaded from The Cancer Genome Atlas (TCGA) database (https://portal. gdc.cancer.gov/). The expression of LINC00238, TMEM106C and ATF3 in HCC tumor tissues and normal tissues were analyzed based on the downloaded data. The overall survival information in this study was downloaded from TCGA database. Survival analysis was performed with the R package 'survival' $(16,17)$ by using the Kaplan-Meier curve method and TANRIC platform. The binding sites between LINC00238 and TMEM106C were predicted by LncTar (www.cuilab. cn/lnctar). Gene Set Enrichment Analysis (GSEA) (18) was performed using three gene set datasets [GO-BP (http://www. geneontology.org), KEGG (http://www.genome.jp/kegg/) and Hallmark gene sets (19)] to analyze the impact of TMEM106C on signaling pathways. The JASPAR (http://jaspar.genereg. net/) and PROMO (alggen.lsi.upc.es/cgi-bin/promo v3/promo/promoinit.cgi?dirDB=TF_8.3) databases were used to analyze the upstream transcription factor of LINC00238.

Patients and samples. A total of 43 human HCC tissues and corresponding normal tissues were collected from 22 male (age range, 32-64 years) and 21 female (age range, 34-70 years) patients with HCC between January 2018 and September 2019. None of the patients received any treatment before surgical resection, including surgery, radiotherapy and chemotherapy. The study was approved by the ethics committee of Qingdao No. 6 People's Hospital [approval no. (2018) 26] and written informed consent was obtained from all patients.

Cell lines and culture. The human normal hepatic cell line MIHA and HCC cell lines Huh7, HLF, SNU-449, MHCC-97h and HCCLM3 were purchased from Mlbio and cultured in DMEM (Gibco; Thermo Fisher Scientific, Inc.) containing antibiotics (Gibco; Thermo Fisher Scientific, Inc.) and 10\% FBS (Gibco; Thermo Fisher Scientific, Inc.), in a humidified atmosphere containing $5 \% \mathrm{CO}_{2}$ at $37^{\circ} \mathrm{C}$.
Cell transfection. LINC00238-overexpressing plasmid pcDNA3.1 (pc-LINC00238), negative control plasmid (pc-NC), short interfering (si)RNAs targeting LINC00238 (30 nM, si-LINC00238\#1, 5'-GAGGATACTTAGGAGAGCTAT-3'; si-LINC00238\#2, 5'-GGAGAGCTATGTATCCTATTT-3'; and si-LINC00238\#3, 5'-GGCTGGAAGTGTTCAGTTAGT-3') and negative control siRNA ( $30 \mathrm{nM}$, si-NC, 5'-GAGGTATG GTATTCGGTCTGA-3'), TMEM106C-overexpressing plasmid pcDNA3.1 (pc-TMEM106C) negative control plasmid (pc-NC), siRNAs targeting ATF3 (30 nM, si-ATF3\#1,5'-GAGCAGTTCG GTGCATATGGT-3'; si-ATF3\#2, 5'-GGGTTACTGGCAGGT TGAACT-3'; and si-ATF3\#3, 5'-GGGAAACAGTTGAGAGGT TAT- $3^{\prime}$ ) and negative control siRNA (30 nM, si-NC, 5'-GCT TGGAAGGTCGGTCATGTA-3'), ATF3-overexpressing plasmid pcDNA3.1 (pc-ATF3) negative control plasmid (pc-NC) were purchased from Shanghai GenePharma Co., Ltd. The transfection was performed by Lipofectamine ${ }^{\circledR} 3000$ (Invitrogen; Thermo Fisher Scientific, Inc.) according to the manufacturer's instructions. The cells were collected after transfection for $48 \mathrm{~h}$.

Reverse transcription-quantitative (RT-q) PCR. Total RNA was extracted from tissues and cells $\left(1 \times 10^{6}\right)$ using TRIzol reagent (Thermo Fisher Scientific, Inc.). The PrimeScript RT reagent kit (Takara Bio, Inc.) was used to synthesize cDNA. qPCR was carried out using SYBR Premix Ex Taq II kit (Takara Bio, Inc.) according to the manufacturer's protocol. The reaction conditions were as follows: $95^{\circ} \mathrm{C}$ for $5 \mathrm{~min}$, followed by 40 cycles of $95^{\circ} \mathrm{C}$ for $15 \mathrm{sec}$ and $60^{\circ} \mathrm{C}$ for $30 \mathrm{sec}$. GAPDH was used as the internal control for mRNA expression. Relative gene expression levels were calculated using the $2^{-\Delta \Delta \mathrm{Cq}}$ method (20). All experiments were performed in triplicate. The primers used in the study were: LINC00238: 5'-TTTGCAGAGTGGGTGCTAGG-3' (forward) and 5'-TGCTTCATCTGGCAATGACCT-3' (reverse); Transmembrane Protein 106C (TMEM106C): 5'-GCCTGT CCAGCCAGATTCAG-3' (forward) and 5'-TTCCTACAGCC CCCTACTCT-3' (reverse); activating transcription factor 3 (ATF3): 5'-AGGTTTGCCATCCAGAACAA-3' (forward) and 5'-AGGCACTCCGTCTTCTCCTT-3' (reverse); GAPDH: 5'-CTGGGCTACACTGAGCACC-3' (forward) 5'-AGTGGT CGTTGAGGGCAATG-3' (reverse).

Cell proliferation assay. HCC cells were trypsinized and seeded into 96 -well culture plates (4,000 cells/well). The cells were harvested at $24,48,72$ and $96 \mathrm{~h}$ to detect proliferation using the 3-(4,5-dimethylthiazol-2-yl)-5-(3-carboxym ethoxyphenyl)-2-(4-sulfophenyl)-2H-tetrazolium (MTS) Cell Proliferation Colorimetric Assay kit (AmyJet Scientific, Inc.) and the absorption was detected at a wavelength of $490 \mathrm{~nm}$.

Cell apoptosis assay. The apoptosis of HCC cells was evaluated using Annexin V/PI apoptosis-detection kit (Invitrogen; Thermo Fisher Scientific, Inc.). HCC cells were washed with Annexin V-binding buffer (Sigma-Aldrich; Merck KGaA), followed by incubation in binding buffer containing Annexin V-FITC $(25 \mu \mathrm{g} / \mathrm{ml})$ and PI $(25 \mu \mathrm{g} / \mathrm{ml})$ in the dark for $10 \mathrm{~min}$ at room temperature. Apoptotic cells (early + late apoptotic cells) were analyzed using a BD Accuri C6 Plus flow cytometer and FACSDiva software (version 6.13; BD Biosciences). 
Wound healing assay. HCC cells were seeded into 6-well plates and incubated to achieve $\geq 90 \%$ confluence. The monolayer cells were scratched with a $10-\mu 1$ sterile micropipette tip and cellular debris was removed by washing with PBS. Then, cells were incubated in serum-free medium with proliferation inhibitor mitomycin $\mathrm{C}(10 \mu \mathrm{M}$; MedChemExpress $)$ at $37^{\circ} \mathrm{C}$. Cell migration was observed at 0 and $48 \mathrm{~h}$ under a light microscope (magnification, x100, Olympus Corporation) and analyzed with ImageJ version 1.49 software (NIH). All experiments were performed in triplicate.

Transwell assay. The invasion of HCC cells was detected by Transwell assay. The upper chambers ( $8 \mu \mathrm{m}$ pore size) were coated with Matrigel at $37^{\circ} \mathrm{C}$ for $30 \mathrm{~min}$. Cells ( $1 \times 10^{4}$ cells/well) were seeded into the upper chamber with serum-free DMEM. DMEM containing 10\% FBS was added to the lower chambers. The chambers were incubated for $48 \mathrm{~h}$ at $37^{\circ} \mathrm{C}$. Subsequently, the noninvasive cells were removed with a cotton swab and the invading cells were fixed with methanol. The cells were then stained with crystal violet at room temperature for 15 min. The number of invasive cells was observed under a light microscope (magnification, x200; Olympus Corporation).

Western blotting. HCC cells $\left(1 \times 10^{6}\right)$ were lysed with RIPA buffer (Beyotime, Shanghai, China) to obtain total protein, centrifuged for $10 \mathrm{~min}$ at $14,000 \mathrm{x} \mathrm{g}$ and the supernatant was collected. The protein concentration was determined using a BCA kit (Beijing Solarbio Science \& Technology Co., Ltd.). Subsequently, the proteins $(20 \mu \mathrm{g})$ were separated by $10 \%$ SDS-PAGE and transferred onto PVDF membranes. The membranes were incubated with $5 \%$ non-fat dry milk for $1 \mathrm{~h}$ at room temperature and then incubated with primary antibodies against TMEM106C (1:500; cat. no. PA5-67816; Thermo Fisher Scientific, Inc.), ATF3 (1:1,000; cat. no. 18665S; Cell Signaling Technology, Inc.), caspase-7 (CASP7) (1:1,000; cat. no. 12827S; Cell Signaling Technology, Inc.), tissue inhibitor of metalloproteinase 2 (TIMP2) (1:1,000; cat. no. 5738S; Cell Signaling Technology, Inc.), programmed cell death 4 (PDCD4) (1:1,000; cat. no. 9535S; Cell Signaling Technology, Inc.) and GAPDH (1:1,000; cat. no. 5174S; Cell Signaling Technology, Inc.) overnight at $4^{\circ} \mathrm{C}$, followed by incubation with the Goat anti-Rabbit IgG Secondary Antibody (1:5,000; cat. no. 31466; Thermo Fisher Scientific, Inc.) at room temperature. GAPDH served as the internal reference. The bands were visualized using enhanced chemiluminescence (ECL) detection reagents (Amersham Biosciences; Cytiva). Blots were semi-quantitatively analyzed using ImageJ software (version 2.0; National Institutes of Health).

RNA immunoprecipitation (RIP) assay. To explore the binding relationship between LINC00238 and TMEM106C, the RIP assay was performed using Magna RIP kit (EMD Millipore) according the manufacturer's protocols. HCC cells were lysed with RIPA lysis buffer (EMD Millipore) and then incubated with anti-TMEM106C-coated Dynabeads Protein G (cat. no. PA5-61466; Thermo Fisher Scientific, Inc.) beads at $4^{\circ} \mathrm{C}$. IgG (cat. no. ab182931; Abcam) was used as the control. The beads were washed with PBS and incubated with TRIzol (Thermo Fisher Scientific, Inc.) for $30 \mathrm{~min}$ at $55^{\circ} \mathrm{C}$. The levels of LINC00238 and TMEM106C were measured using RT-qPCR.
Statistical analysis. Data are shown as means \pm SD and were analyzed using SPSS 23.0 (IBM Corp.) and GraphPad Prism 7.0 (GraphPad Software, Inc.). Comparisons between two groups were analyzed using unpaired Student's t-test and the comparisons among multiple groups were analyzed by one-way ANOVA followed by Tukey's post hoc test. Pearson's correlation analysis was used to assess the correlation among LINC00238, TMEM106C and ATF3 expression. All experiments were performed in triplicate.

\section{Results}

LINC00238 is downregulated in HCC. The online platform GEPIA was used to analyze the differentially expressed lncRNAs in HCC and the results suggested that LINC00238 was downregulated in HCC. The online analysis based on TCGA suggested that LINC00238 levels in HCC tissues were markedly lower compared with those in normal tissues (Fig. 1A) and that LINC00238 levels was positively correlated with the overall survival of patients with HCC (Fig. 1B). LINC00238 expression was measured in $\mathrm{HCC}$ tissues and corresponding normal tissues using RT-qPCR. The data showed that LINC00238 was significantly downregulated in HCC tumor tissues (Fig. 1C). High levels of LINC00238 expression were associated with local TNM stage (I/II; Fig. 1D) and non-metastasis (Fig. 1E). LINC00238 expression in human normal hepatic cell line MIHA and HCC cell lines (Huh7, HLF, SNU-449, MHCC-97h and HCCLM3) was measured using RT-qPCR. The results indicated that LINC00238 expression in HCC cell lines was markedly decreased compared with that in the normal hepatic cell line (Fig. 1F). HCCLM3 and MHCC-97h cell lines were selected for further study because of the low LINC00238 expression in these cells.

LINC00238 overexpression inhibits cell proliferation, migration and invasion of HCC cells. The LINC00238 overexpressing plasmid pc-LINC00238 was transfected into HCCLM3 and MHCC-97h cells to detect the influence of LINC00238 on the biological function of HCC cells. As shown in Fig. 2A, LINC00238 expression was significantly increased in the pc-LINC00238 group compared with that in pc-NC group. The proliferation of HCC cells was measured using the MTS assay. The data suggested that LINC00238 overexpression suppressed the proliferation ability of HCCLM3 and MHCC-97h cells (Fig. 2B). Flow cytometry results revealed that the apoptotic rates of HCCLM3 and MHCC-97h cells were significantly increased after LINC00238 overexpression (Fig. 2C). The migration and invasion capacity of HCC cells was detected by wound healing and Transwell assays, respectively. The results in Fig. 2D showed that LINC00238 overexpression markedly decreased the migration capacity of HCC cells. Additionally, the number of invasive cells in the pc-LINC00238 group was significantly reduced compared with that in the pc-NC group (Fig. 2E).

LINC00238 negatively regulates the expression of TMEM106C in HCC. A recent study showed that TMEM106C is highly expressed in HCC and serves an important role in HCC progression (14). LncTar was performed to determine whether there is a targeted relationship between LINC00238 and TMEM106C (21) 

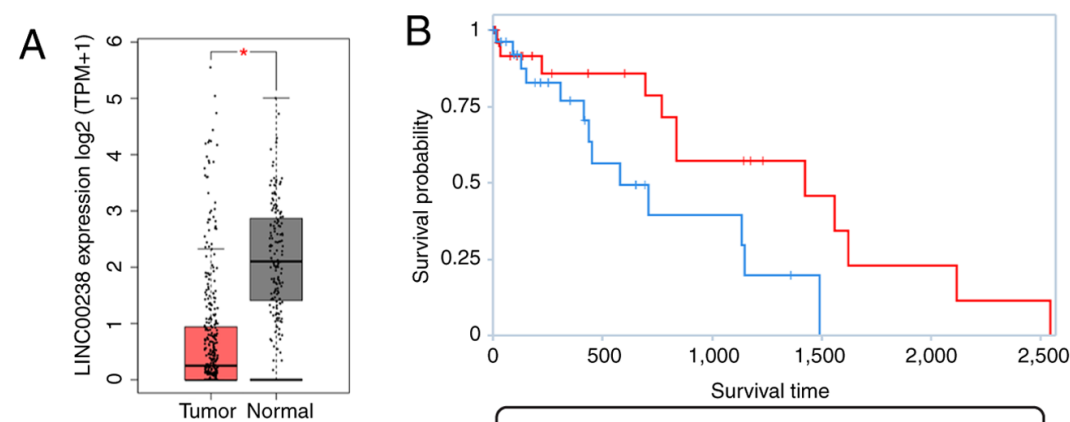
$(\operatorname{num}(\mathrm{T})=369 ; \operatorname{num}(\mathrm{N})=160)$
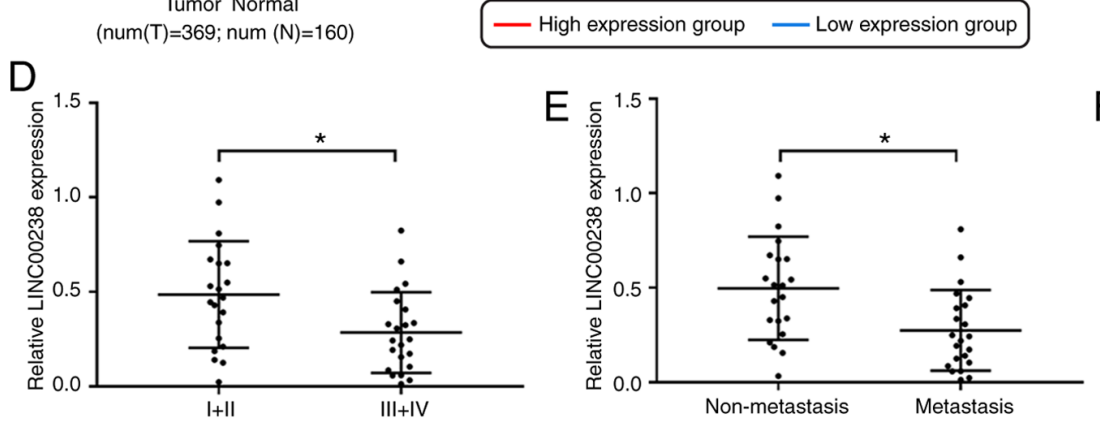
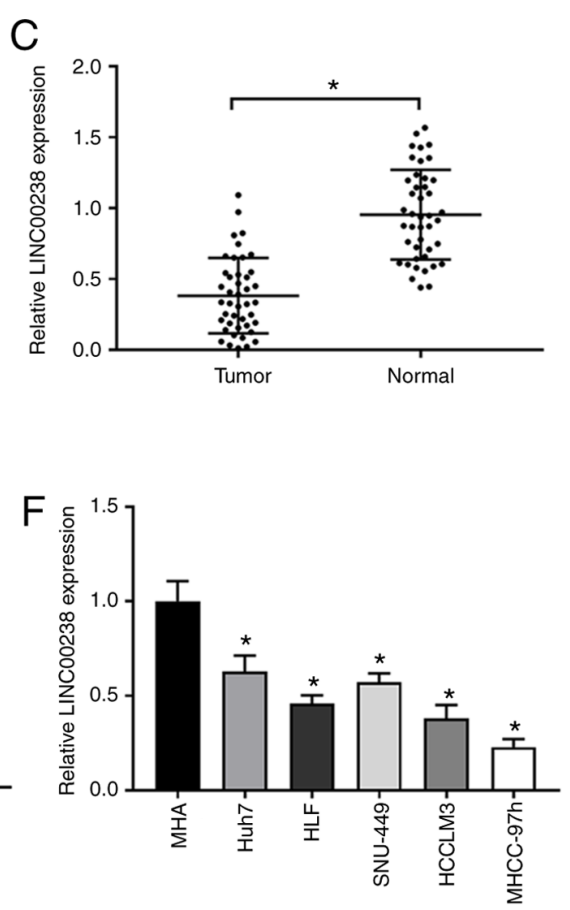

Figure 1. LINC00238 is downregulated in HCC. (A) Scatter plots comparing LINC00238 expression in HCC samples (n=396) and normal tissue samples $(\mathrm{n}=160)$ showed that LINC00238 exhibited low expression in HCC tumor samples. (B) Kaplan-Meier analysis indicated a correlation between low LINC00238 and poor overall survival in patients with HCC. The graph was conducted by TANRIC platform. (C) RT-qPCR data showed that LINC00238 was downregulated in HCC tumor tissues compared with normal tissues. (D) RT-qPCR data showed that low expression level of LINC00238 in patients with HCC was associated with early stage (I/II) disease of HCC. (E) RT-qPCR data showed that the low expression levels of LINC00238 in patients with HCC was associated with non-metastasis. (F) RT-qPCR data showed that LINC00238 was downregulated in HCC cell lines (Huh7, HLF, SNU-449, MHCC-97h and HCCLM3) compared with human normal hepatic cell line MIHA. "P<0.05. LINC, long intergenic non-protein coding RNA; HCC, hepatocellular carcinoma; RT-qPCR, reverse transcription-quantitative PCR.

and the results (Fig. 3A) suggested that TMEM106C was the target gene of LINC00238. The target relationship between LINC00238 and TMEM106C was verified using RIP assay. The data suggested that LINC00238 expression was significantly increased in TMEM106C immunoprecipitates in HCCLM3 and MHCC-97h cells compared with that the Anti-IgG group, suggesting that TMEN106C could couple with LINC00238 directly (Fig. 3B). The protein levels of TMEM106 in HCC cells after LINC00238 overexpression was evaluated using western blotting. The data showed that LINC00238 overexpression significantly reduced TMEM106 expression (Fig. 3C). The online data based on TCGA suggested that TMEM106C is more highly expressed in HCC tumor tissues than in normal tissues (Fig. 3D) and a high level of TMEM106C expression is associated with poor overall survival (Fig. 3E). There was a negative correlation between LINC00238 and TMEM106C expression in HCC (Fig. 3F). TMEM106C expression was detected with RT-qPCR and the correlation between LINC00238 and TMEM106C expression analyzed. The results indicated that TMEM106C expression was significantly upregulated in tumor tissues (Fig. 3G) and negatively correlated with LINC00238 expression (Fig. $3 \mathrm{H}$ ).

To investigate whether LINC00238 served its role through TMEM106C, pc-LINC00238 and pc-TMEM106C were co-transfected into HCCLM3 and MHCC-97h cells to detect the influence of LINC00238 and TMEM106C on HCC progression. As shown in Fig. 3I, the TMEM106C expression was significantly increased by TMEM106C overexpression. The proliferation ability of HCC cells was markedly enhanced by TMEM106C overexpression (Fig. 3J). Additionally, the capacity of invasion (Fig. 3K) was significantly higher in the p-TMEM106C group than that in the pc-NC group. In addition, the inhibitory effect of LINC00238 overexpression on the proliferation and invasion of $\mathrm{HCC}$ cells was eliminated with TMEM106C overexpression.

TMEM106Cregulates the apoptosis pathway and LINC00238 is transcriptionally regulated by ATF3 in HCC. The present study performed GESA analysis of the gene sequences of TMEM106C in HCC cells (18). The results showed that TMEM106C suppressed the apoptosis signaling pathway (Fig. 4A). The protein expression of genes enriched in the apoptosis signaling pathway was then detected. Western blot analysis showed that the protein expression of capsase-7 (CASP7), TIMP2, PDCD4 and ATF3 was significantly increased by LINC00238 overexpression and decreased by TMEM106C overexpression (Fig. 4B). The inhibitory effect of TMEM106C on protein expression of ASP7, TIMP2, PDCD4 and ATF3 in HCC cells was eliminated by LINC00238 overexpression (Fig. 4B).

To investigate the potential mechanism of LINC00238 in HCC, the JASPAR and PROMO databases were analyzed and ATF3 was identified as the upstream transcription factor of LINC00238 (Fig. 4C and D). The regulatory effect of ATF3 on LINC00238 expression was detected after ATF3 siRNAs (si-ATF3\#1, si-ATF3\#2 and si-ATF3\#3) were transfected into HCC cells. As shown in Fig. 4E, ATF3 levels were significantly decreased in the si-ATF3 groups. The expression of LINC00238 was markedly suppressed in the si-ATF3 

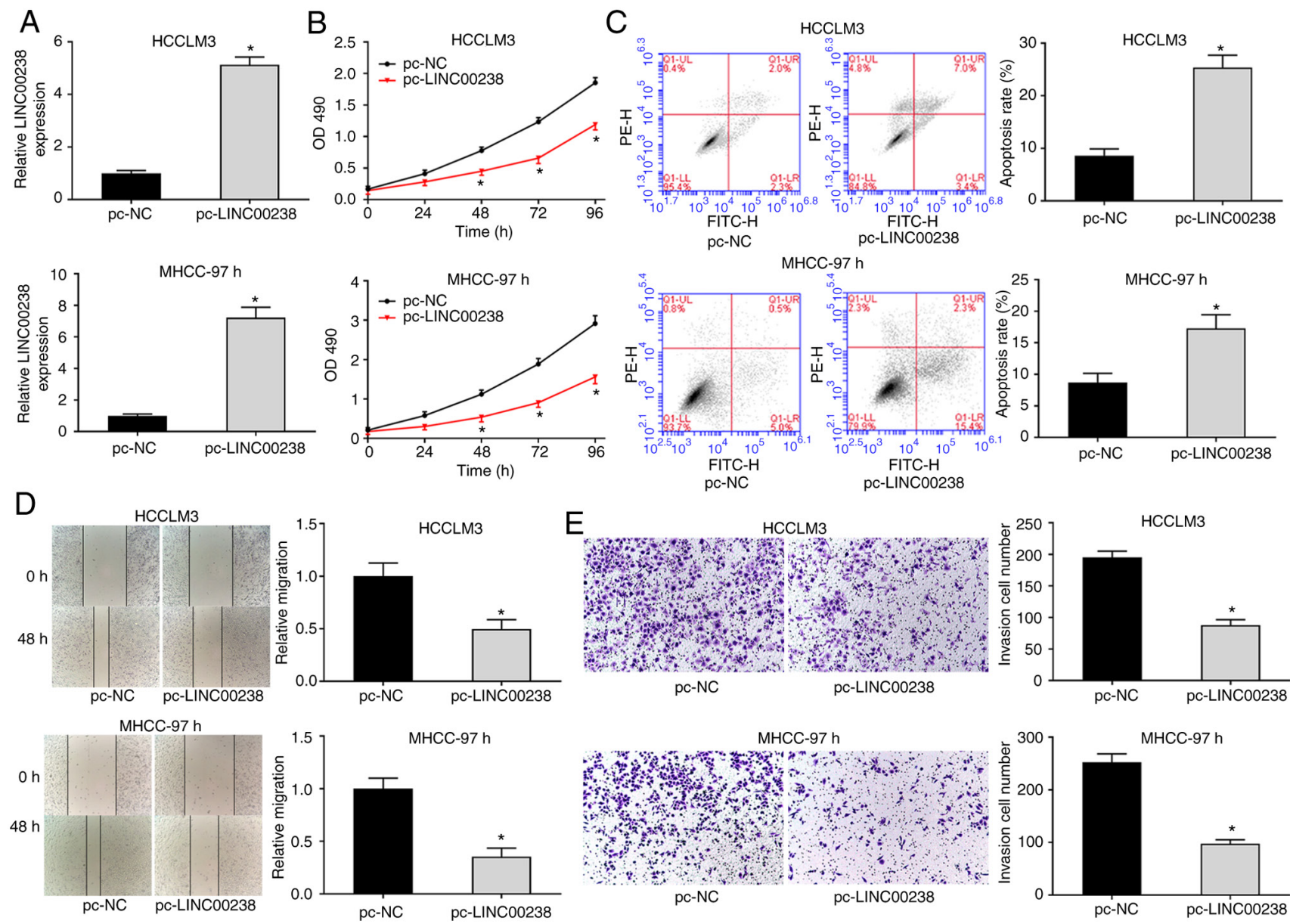

Figure 2. LINC00238 overexpression inhibits cell proliferation, migration and invasion of HCC. (A) RT-qPCR data showed that LINC00238 expression in HCCLM3 and MHCC-97h cells was significantly increased by LINC00238 overexpression. (B) Data from MTS assay showed that the proliferation ability of HCCLM3 and MHCC-97h cells was significantly decreased by LINC00238 overexpression. (C) The flow cytometry data showed that the apoptotic rates of HCCLM3 and MHCC-97h cells were increased by LINC00238 overexpression. (D) Wound healing assay showed that the migration capacity of HCCLM3 and MHCC-97h cells was suppressed by LLINC00238 overexpression (magnification, x100). (E) The data of Transwell assay showed that the invasion capacity of HCCLM3 and MHCC-97h cells was suppressed by LLINC00238 overexpression. ${ }^{*} \mathrm{P}<0.05$ vs. pc-NC group. LINC, long intergenic non-protein coding RNA; HCC, hepatocellular carcinoma; RT-qPCR, reverse transcription-quantitative PCR; pc-NC, negative control plasmid.

groups compared with that in the si-NC group (Fig. 4F). ATF3 expression in HCC tissues and corresponding normal tissues was measured using RT-qPCR. The results indicated that ATF3 expression in tumor tissues was lower compared with that in normal tissues (Fig. 4G). Online data based on TCGA suggested that ATF3 was expressed at low levels in HCC tumor tissues (Fig. 4H) and low levels of ATF3 expression were associated with poor overall survival (Fig. 4I). Furthermore, there was a positive correlation between ATF3 and LINC00238 and a negative correlation between ATF3 and TMEM106C in HCC (Fig. 4J).

ATF3 overexpression reverses the promoting effect of LINC00238 silencing on HCC progression. The si-LINC00238 and ATF3-overexpressing plasmid were co-transfected into HCC cells to explore the regulatory effect of ATF3 on LINC00238 in HCC progression. As shown in Fig. 5A, LINC00238 expression was significantly decreased by LINC00238 silencing. The ATF3 expression was markedly increased by ATF3 overexpression (Fig. 5B). The MTS data showed that LINC00238 silencing enhanced cell proliferation, whereas ATF3 overexpression reduced the proliferation of
HCC cells (Fig. 5C). Compared with the si-LINC00238 group, the proliferation ability of the si-LINC00238+pc-ATF3 group was significantly decreased (Fig. 5C). Apoptosis of HCC cells was measured using flow cytometry. The data suggested that apoptosis of HCC cells was markedly decreased by LINC00238 silencing and increased by ATF3 overexpression (Fig. 5D). The inhibition of LINC00238 silencing on HCC cell apoptosis was eliminated by ATF3 overexpression (Fig. 5D). Additionally, the migration and invasion capacity of HCC cells following co-transfection with si-LINC00238 and pc-ATF3 were measured. The data suggested that the capacity of migration and invasion of HCC cells was significantly enhanced by LINC00238 silencing and reduced by ATF3 overexpression (Fig. 5E and F). Besides, ATF3 overexpression reversed the accelerating effect of LINC00238 silencing on the migration and invasion of HCC cells (Fig. 5E and F).

\section{Discussion}

HCC is one of the commonest cancers with high mortality rates worldwide. High rates of metastasis and postoperative recurrence lead to high mortality rate in patients with HCC (3). 


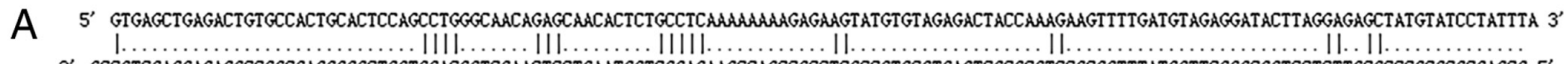

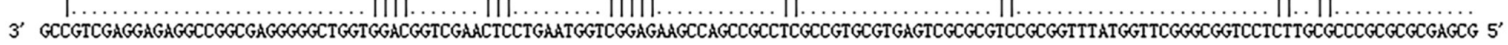
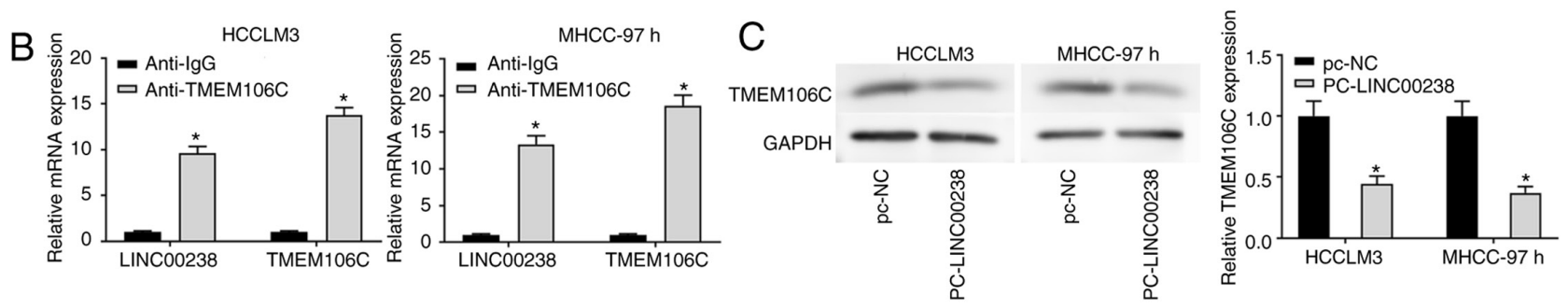

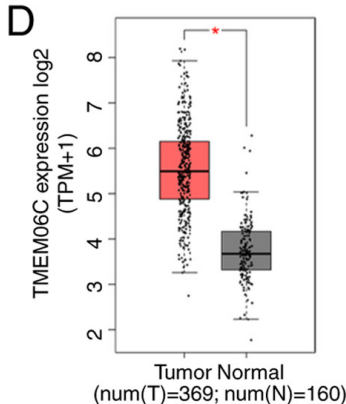

G
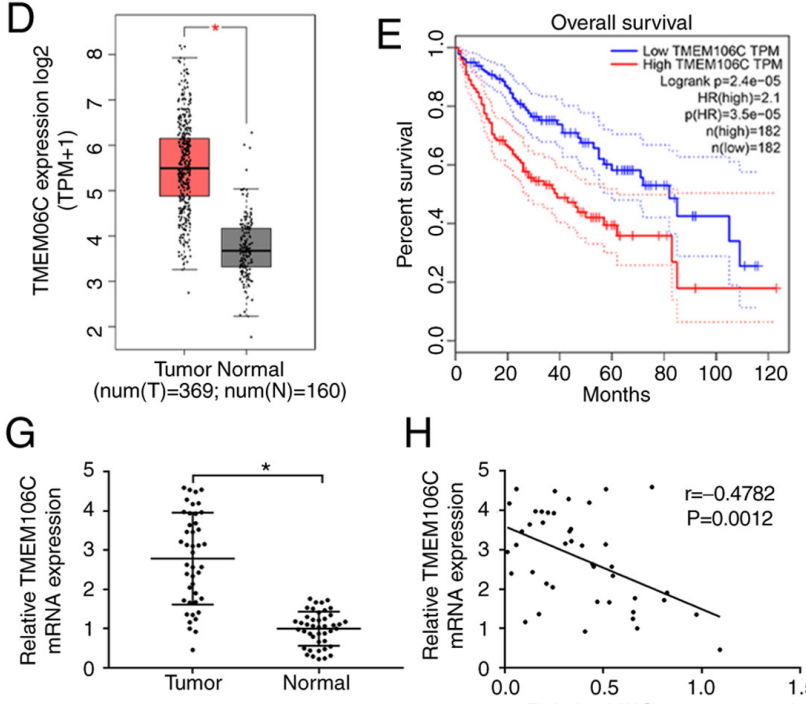

$\mathrm{H}$

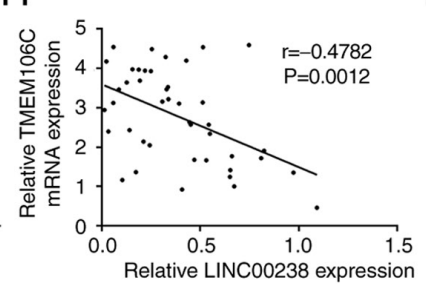

F

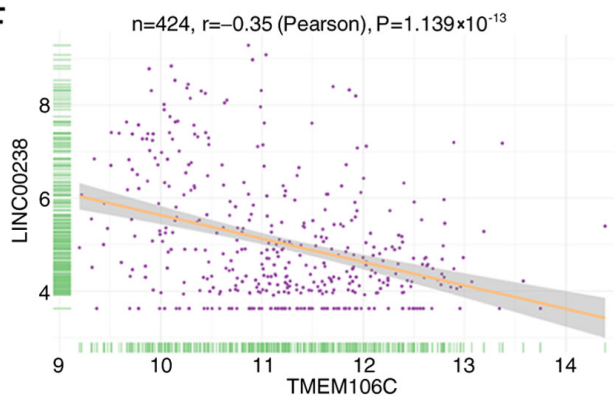

I

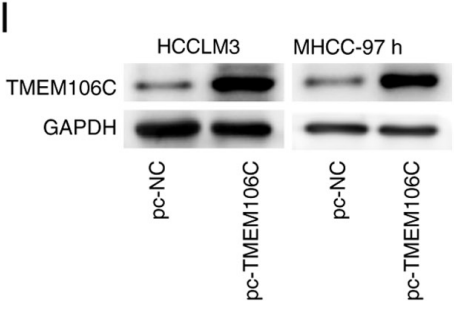

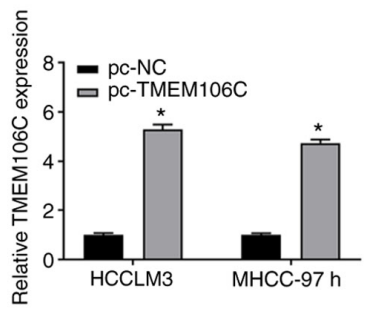
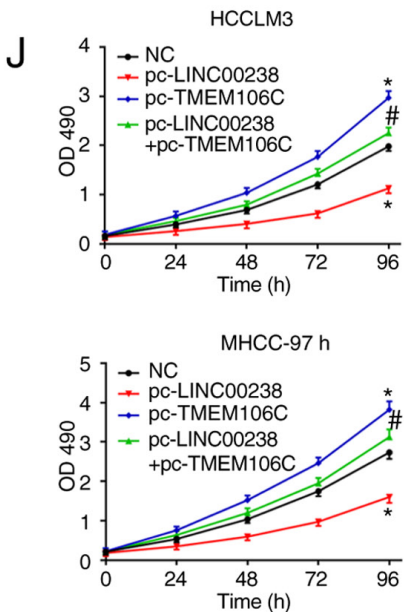
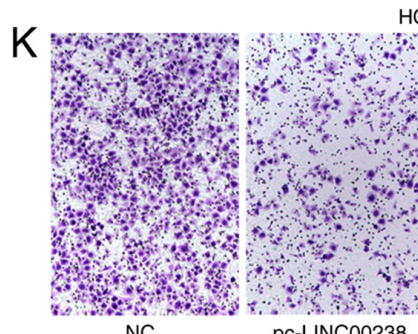

HCCLM3

NC

pc-LINC0023

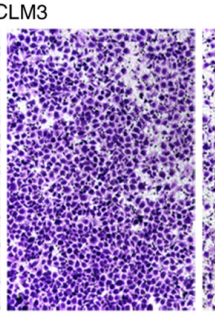

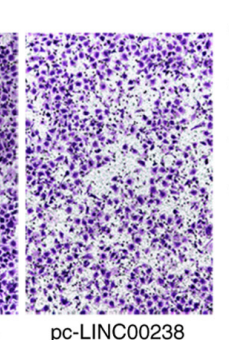

pc-LINC00238 +pc-TMEM106C

MHCC-97 h

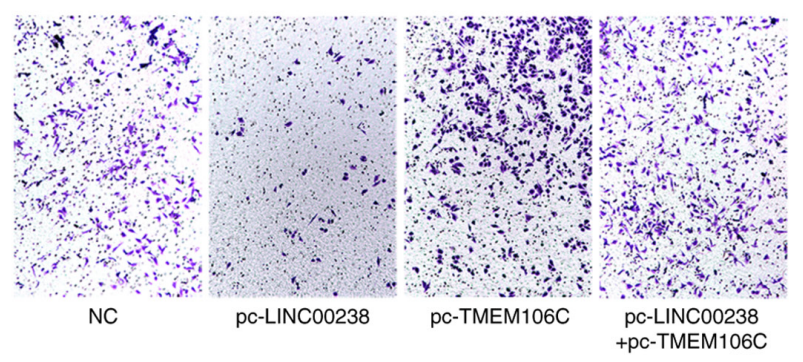

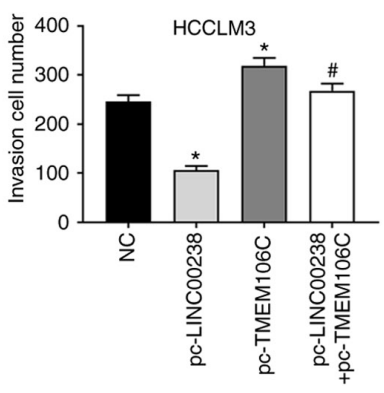

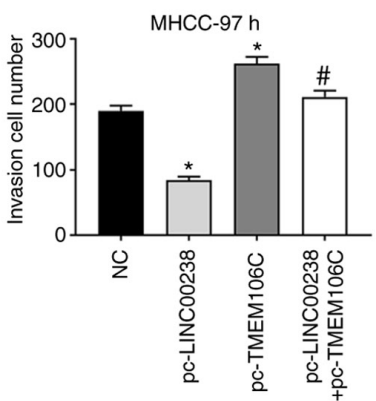

Figure 3. LINC00238 negatively regulates the expression of TMEM106C in HCC. (A) The predicted result of LncTar showed that MEM106C was a target gene of LINC00238. A dot in the graph indicates there is a paired of bases in the two sequences. A line in the graph indicates there is a paired of complementary bases in the two sequences. (B) The data of RNA immunoprecipitation assay showed that TMEM106C was a target of LINC00238 in HCCLM3 and MHCC-97h cells. (C) The western blot results showed that protein expression of TMEM106C in HCC cells was decreased by LINC00238 overexpression. (D) Scatter plots comparing TMEM106C expression in HCC samples $(n=396)$ and normal tissue samples $(n=160)$ showed that TMEM106C was upregulated in HCC tumor samples compared with normal samples. (E) Kaplan-Meier analysis indicated a correlation between high TMEM106 and poor overall survival rates in patients with HCC. (F) Correlation analysis between LINC00238 and TMEM106C expression showed that the LINC00238 expression was negatively correlated with TMEM106C expression. (G) RT-qPCR data showed that TMEM106C expression was upregulated in HCC tumor tissues compared with corresponding normal tissues. "P<0.05. (H) Pearson's correlation analysis of the relationship between LINC00238 and TMEM106C in patients with HCC showed that the LINC00238 expression was negatively correlated with TMEM106C expression. (I) The results of western blotting showed that the expression of TMEM106C protein was markedly increased by TMEM106C overexpression. (J) MTS assay showed that TMEM106C overexpression increased the proliferation ability of HCCLM3 and MHCC-97h cells and reversed the inhibitory effect of LINC00238 overexpression on proliferation of HCC cells (K) Transwell assay showed that TMEM106C overexpression enhanced the invasion capacity of HCCLM3 and MHCC-97h cells and reversed the inhibitory effect of LINC00238 overexpression on invasion of HCC cells (magnification, $\mathrm{x} 200$ ). ${ }^{*} \mathrm{P}<0.05$ vs. pc-NC group; ${ }^{\sharp} \mathrm{P}<0.05$ vs. pc-LINC00238 group. LINC, long intergenic non-protein coding RNA; HCC, hepatocellular carcinoma; TMEM106C, transmembrane protein 106C; pc-, plasmid; NC, negative control. 
A

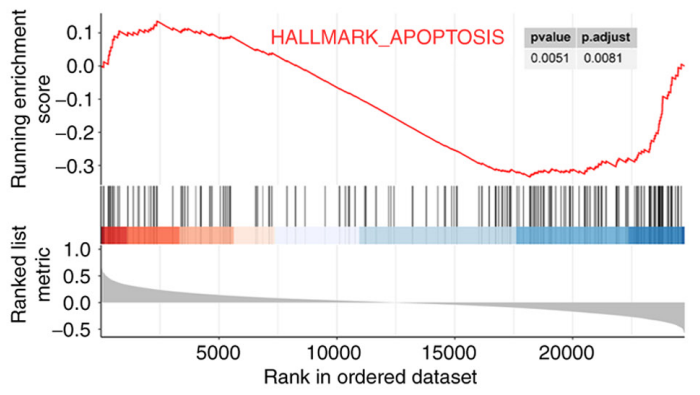

C

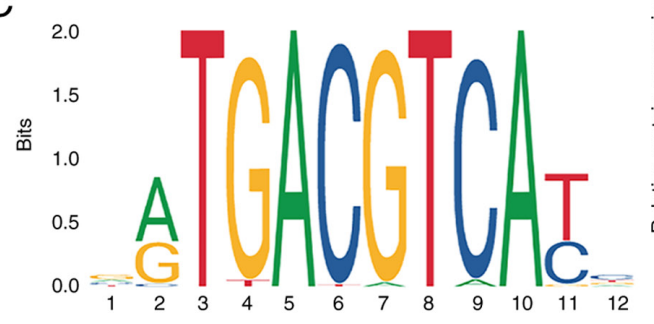

D

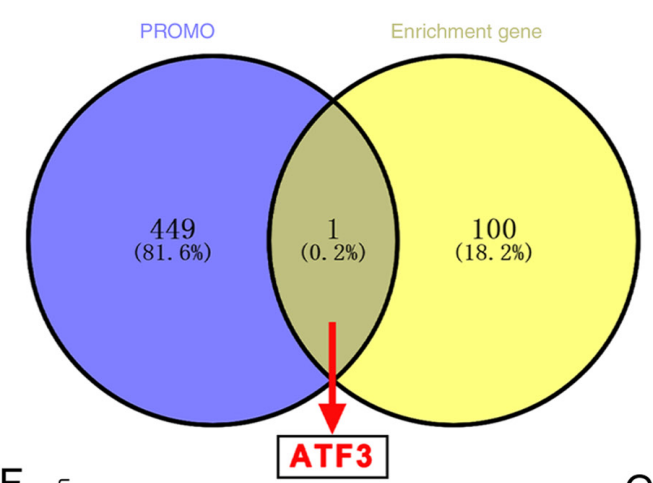

F

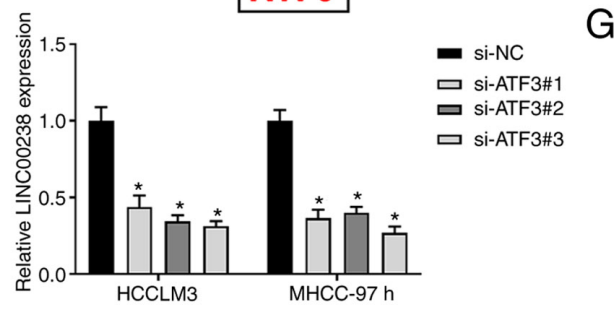

B
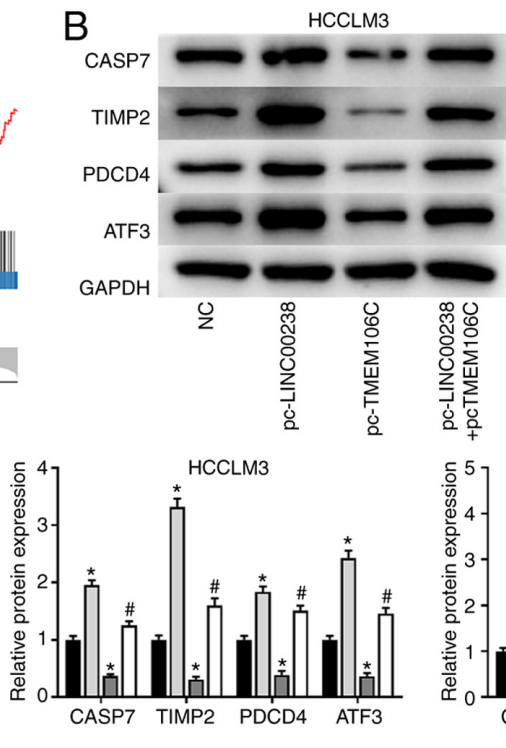

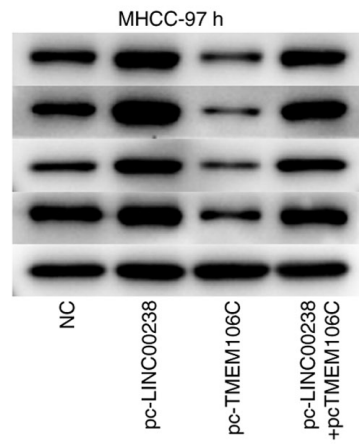

MHCC-97 h

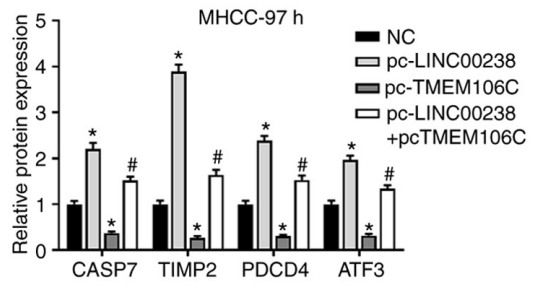

E

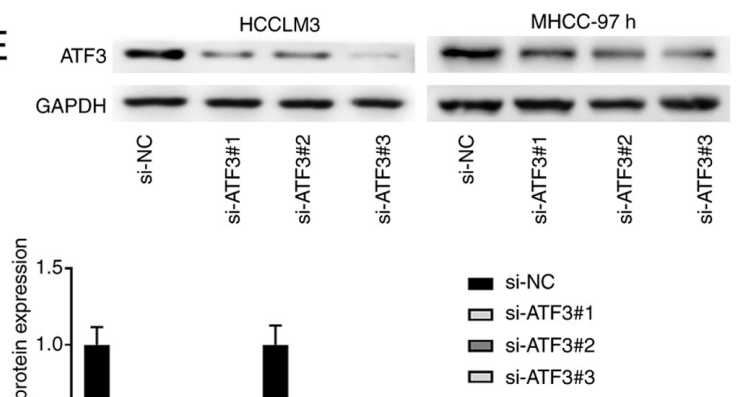

$\mathrm{H}$
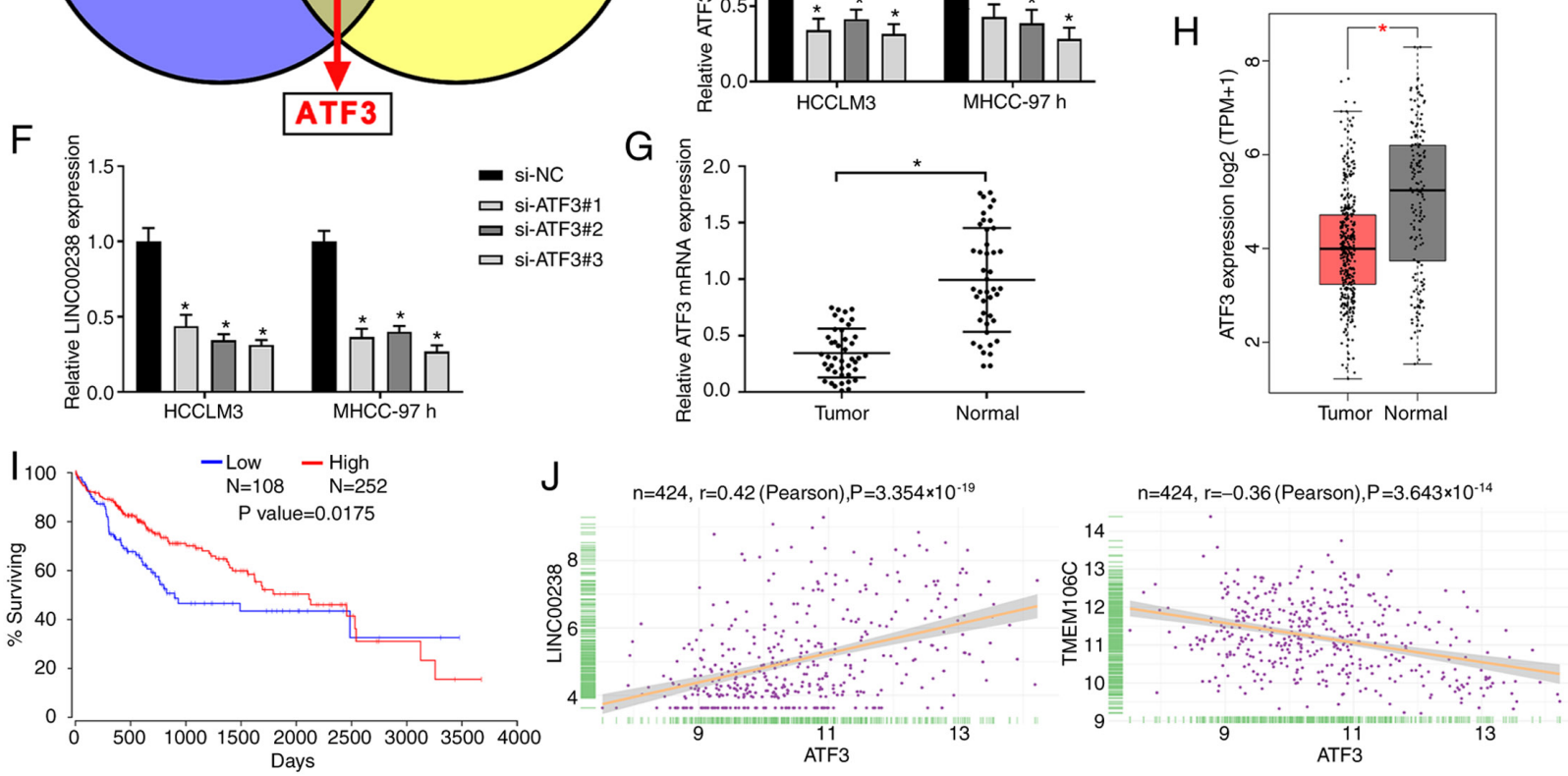

Figure 4. TMEM106C regulates apoptosis pathway and LINC00238 is transcriptionally regulated by ATF3 in HCC. (A) The Enriched KEGG pathway analysis showed that TMEM106C suppressed the apoptosis signaling pathway. (B) The results of western blotting showed that protein expression of ASP7, TIMP2, PDCD4 and ATF3 in HCCLM3 and MHCC-97h cells was increased by LINC00238 overexpression while decreased by TMEM1106C overexpression. "P $<0.05$ vs. NC group; ${ }^{\text {P }}<0.05$ vs. pc-LINC00238 group. (C) Recognition motif of ATF3 from the JASPAR database. (D) Venn diagram showing ATF3 is upstream transcription factor of LINC00238 of LINC00958 predicted by PROMO and KEGG. (E) The results of western blotting showed that ATF3 protein expression in HCCLM3 and MHCC-97h cells was decreased by ATF3 siRNAs. (F) The results of RT-qPCR showed that LINC00238 expression in HCCLM3 and MHCC-97h cells was decreased by ATF3 siRNAs. (G) The results of RT-qPCR showed that ATF3 expression was significantly lower in HCC tissues than that in corresponding normal tissues. (H) Scatter plots comparing ATF3 expression in HCC samples $(n=396)$ and normal tissue samples ( $\mathrm{n}=160)$ showed that ATF3 was downregulated in HCC tumor samples compared with normal samples. (I) Kaplan-Meier analysis indicated an association between high TMEM106 and poor overall survival in patients with HCC. (J) The correlation analysis among ATF3, LINC00238 and TMEM106C showed that the ATF3 expression was positively correlated with LINC00238 and negatively correlated with TMEM106C expression. "P<0.05. TMEM106C, transmembrane protein 106C; LINC, long intergenic non-protein coding RNA; HCC, hepatocellular carcinoma; KEGG, Kyoto Encyclopedia of Genes and Genomes; ATF3, activating transcription factor 3; CASP7, caspase-7; TIMP2, tissue inhibitor of metalloproteinase 2; PDCD4, programmed cell death 4; pc-, plasmid; si, short interfering; RT-qPCR, reverse transcription-quantitative PCR. 

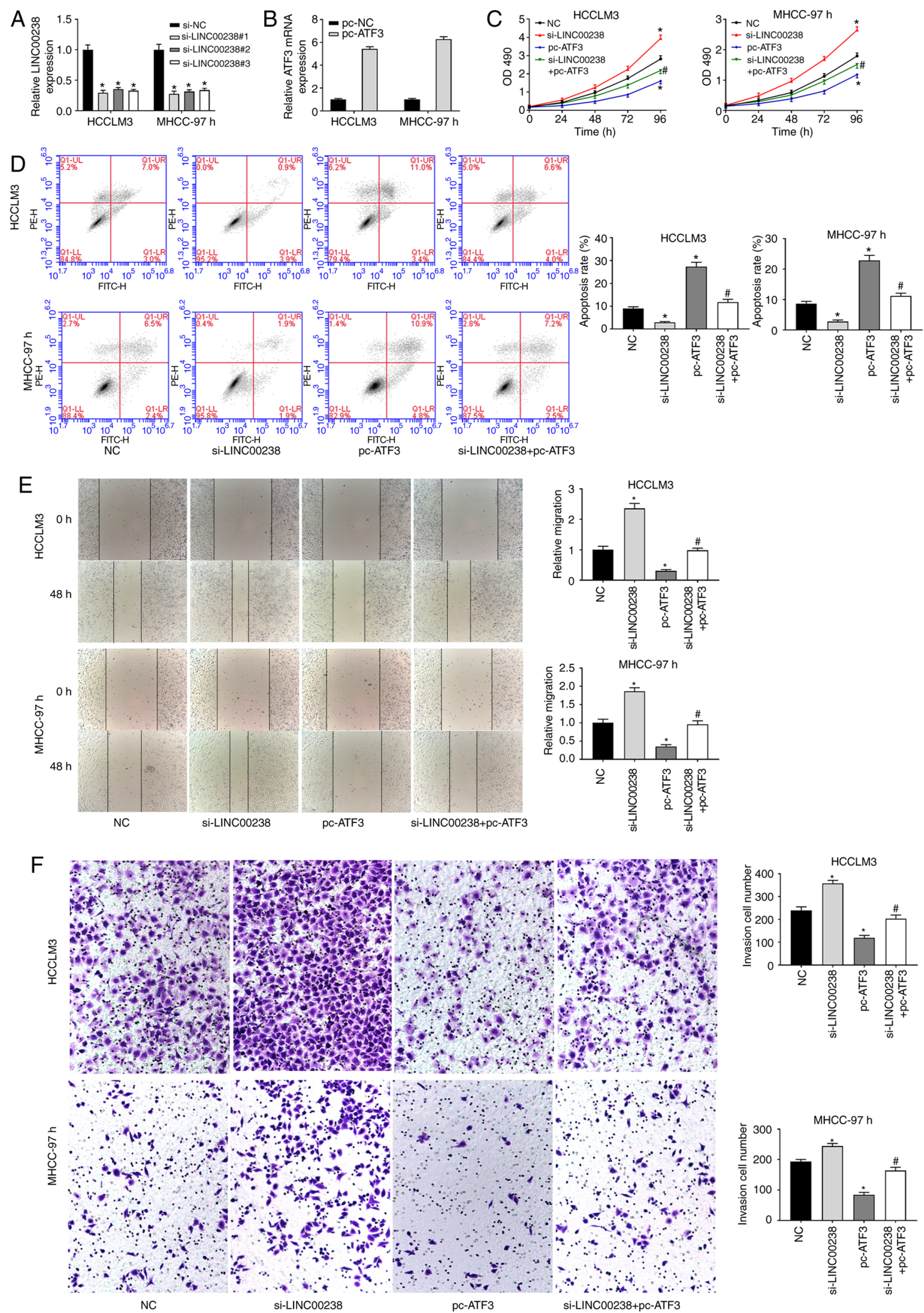

Figure 5. ATF3 overexpression reverses the promoting effect of LINC00238 silencing on the procession of HCC. (A) RT-qPCR showed that LINC00238 expression was significantly decreased by LINC00238 silencing. (B) RT-qPCR showed that ATF3 expression was significantly decreased by ATF3 overexpression. (C) MTS assay showed that ATF3 overexpression reduced the proliferation ability of HCCLM3 and MHCC-97h cells and eliminated the promoted effect of LINC00238 silence on HCC proliferation. (D) Flow cytometry showed that ATF3 overexpression increased the apoptosis of HCCLM3 and MHCC-97h cells and eliminated the inhibitory effect of LINC00238 silence on HCC apoptosis. (E) Wound healing assay showed that ATF3 overexpression inhibited the migration capacity of HCCLM3 and MHCC-97h cells and eliminated the promoted effect of LINC00238 silence on HCC migration (magnification, x100), (F) Transwell assay showed that ATF3 overexpression inhibited the invasion capacity of HCCLM3 and MHCC-97h cells and eliminated the promoted effect of LINC00238 silence on HCC invasion (magnification, $\mathrm{x} 200$ ). ${ }^{\text {"P }}<0.05$ vs. NC group; ${ }^{*} \mathrm{P}<0.05$ vs. si-LINC00238 group. ATF3, activating transcription factor 3 ; LINC, long intergenic non-protein coding RNA; HCC, hepatocellular carcinoma; RT-qPCR, reverse transcription-quantitative PCR. 
The present study found that LINC00238 was downregulated in HCC. Overexpression of LINC00238 suppressed proliferation, migration and invasion of $\mathrm{HCC}$ cells by activating the TMEM106C-mediated apoptosis signaling pathway.Furthermore, it was found that ATF3, a gene enriched in the apoptosis signaling pathway, was the upstream promoter of LINC00238.

Previous studies have suggested that lncRNAs serve a vital role in the initiation and progression of HCC. Mo et al (22) revealed that LINC01287 expression is increased in HCC cells and knockdown of LINC01287 decreases the proliferation and invasion ability of HCC cells. The lncRNA CTC-297N7.9 acts as a tumor suppressor in HCC and low expression of lncRNA CTC-297N7.9 is associated with poor prognosis in patients with HCC (23). In the present study, the expression of LINC00238 was significantly decreased in both HCC tissues and cell lines. High levels of LINC00238 were associated with a good prognosis. Overexpression of LINC00238 inhibited the proliferation, migration and reduced invasion capacity of $\mathrm{HCC}$ cells. These data indicated that LINC00238 acted as a tumor suppressor in HCC and LINC00238 overexpression inhibited HCC progression.

TMEM106C is a member of the TMEM106 family and is expressed at low levels in patients with ankylosing spondylitis (13). Duan et al (24) found that TMEM106C expression is positively related to CENPM and negatively related to DLC-1 in HCC tumor tissues. Luo et al (14) showed that FOXO1 and FOXO3 may be the key target genes of TMEM106C in HCC. In the present study, LINC00238 expression was higher in HCC tissues compared with that in normal tissues. The predicted result of LncTar suggested that TMEM106C was the target of LINC00238. LINC00238 overexpression reduced the protein expression of TMRM106C. TCGA analysis showed that high TMEM106C expression was associated with poor prognosis of HCC and negatively correlated with LINC00238 expression. In addition, TMEM106C overexpression reversed the inhibitory effect of LINC00238 overexpression on the proliferation, migration and invasion of HCC cells. These data suggested that LINC00238 inhibited HCC progression by suppressing TMEM106C expression.

The expression of the ATF3, a member of the ATF family of transcription factors, can be induced by a variety of stress signals (25). Previous studies have demonstrated that ATF3 acts as a tumor inhibitor in cancer. ATF3 dysfunction allows normal cells to be easily transformed by oncogenes (26). For instance, Li et al (27) found that overexpression of ATF3 inhibits the proliferation and migration in esophageal squamous cell carcinoma. The findings of Hackl et al (28) indicated that downregulation of ATF3 promotes the migration capacity of colon cancer cells in vitro and facilitates tumor growth in vivo. Lv et al (29) observed that ATF3 is significantly downregulated in human intrahepatic cholangiocarcinoma with tumor metastasis. The results of the present study were consistent with those of previous studies. The expression of ATF3 was significantly lower in HCC tumor tissues than that in normal tissues. Low ATF3 expression was associated with poor prognosis in patients with HCC. ATF3 overexpression inhibited proliferation, migration and invasion of HCC cells. In addition, it was found that ATF3 was an upstream transcription factor of LINC00238. ATF3 expression was positively correlated to LINC00238 and LINC00238 expression could be reduced by ATF3 silencing. Overexpression of ATF3 reversed the promoting effect of LINC00238 silencing on proliferation, migration and invasion of HCC cells. These results indicated that ATF3 could suppress HCC progression and strengthen the inhibitory effect of LINC00238 on HCC.

Avoidance of apoptosis a major cause of cancer development and progression (30). CASP7 is a member of the caspases family that participates in cervical cancer progression (31). Palmerini et al (32) indicated that CASP7 is downregulated in colon cancer. Proteins encoded by TIMPs are natural inhibitors of matrix metalloproteinases. Peeney et al (33) demonstrated that TIMP2 inhibits the proliferation and EMT progression of triple-negative breast cancer. PDCD4 is a tumor suppressor gene for various cancers and can inhibit cell proliferation, migration and invasion, as well as promote tumor cell apoptosis (34). Hwang et al (35) indicated that overexpression of PDCD4 induces anti-proliferation and apoptosis-induced effects on human lung cancer. In the present study, western blotting data showed that LINC00238 overexpression increased the protein expression of CASP7, TIMP2 and PDCD4. However, the promoting effect of LINC00238 on CASP7, TIMP2 and PDCD4 was eliminated by TMEM106C overexpression. These results suggested that LINC00238 inhibited the proliferation, migration and invasion of HCC cells by upregulating these genes that were enriched in the apoptosis pathway.

In conclusion, LINC00238 was downregulated in HCC tissues and cell lines and served a vital role in restraining HCC progression. Overexpression of LINC00238 decreased TMEM106C expression and increased the expression of CASP7, TIMP2, PDCD4 and ATF3, which are apoptotic pathway genes and thus inhibited the proliferation, migration and invasion of HCC cells. Notably, ATF3 positively regulated the expression of LINC00238, thereby participating in the inhibitory regulation of HCC by LINC00238.

\section{Acknowledgements}

Not applicable.

\section{Funding}

No funding was received.

\section{Availability of data and materials}

The datasets used and/or analyzed during the current study are available from the corresponding author on reasonable request.

\section{Authors' contributions}

CJ and FL designed the experiments, MY, JD and SF collected data. JL and SS analyzed the data and wrote the manuscript. FL and CJ confirm the authenticity of all the raw data. All authors reviewed and approved the final manuscript.

\section{Ethics approval and consent to participate}

The study was approved by the ethics committee of Qingdao No. 6 People's Hospital [Approval no. (2018) 26] and written informed consent was obtained from all patients. 


\section{Patient consent for publication}

Not applicable.

\section{Competing interests}

The authors declare that they have no competing interests.

\section{References}

1. Bray F, Ferlay J, Soerjomataram I, Siegel RL, Torre LA and Jemal A: Global cancer statistics 2018: GLOBOCAN estimates of incidence and mortality worldwide for 36 cancers in 185 countries. CA Cancer J Clin 68: 394-424, 2018.

2. Bellissimo F, Pinzone MR, Cacopardo B and Nunnari G: Diagnostic and therapeutic management of hepatocellular carcinoma. World J Gastroenterol 21: 12003-12021, 2015.

3. Cheng D, Deng J, Zhang B, He X, Meng Z, Li G, Ye H, Zheng S, Wei L, Deng X, et al: LncRNA HOTAIR epigenetically suppresses miR-122 expression in hepatocellular carcinoma via DNA methylation. EBioMedicine 36: 159-170, 2018.

4. Lou W, Liu J, Gao Y, Zhong G, Ding B, Xu L and Fan W: MicroRNA regulation of liver cancer stem cells. Am J Cancer Res 8: 1126-1141, 2018.

5. Huang Z, Zhou JK, Peng Y, He W and Huang C: The role of long noncoding RNAs in hepatocellular carcinoma. Mol Cancer19: 77, 2020.

6. Gong D, Feng PC, Ke X-F, Kuang HL, Pan LL, Ye Q and Wu JB: Silencing long non-coding RNA LINC01224 inhibits hepatocellular carcinoma progression via MicroRNA-330-5p-induced inhibition of CHEK1. Mol Ther Nucleic Acids 19: 482-497, 2020.

7. Jia M, Jiang L, Wang YD, Huang JZ, Yu M and Xue HZ: LincRNA-p21 inhibits invasion and metastasis of hepatocellular carcinoma through Notch signaling induced epithelial-mesenchymal transition. Hepatol Res 46: 1137-1144, 2016.

8. Liu F, Yuan JH, Huang JF, Yang F, Wang TT, Ma JZ, Zhang L, Zhou CC, Wang F, Yu J, et al: Long noncoding RNA FTX inhibits hepatocellular carcinoma proliferation and metastasis by binding MCM2 and miR-374a. Oncogene 35: 5422-5434, 2016.

9. Wang CZ, Yan GX, Dong DS, Xin H and Liu ZY: LncRNA-ATB promotes autophagy by activating Yes-associated protein and inducing autophagy-related protein 5 expression in hepatocellular carcinoma. World J Gastroenterol 25: 5310-5322, 2019.

10. Marx S, Dal Maso T, Chen JW, Bury M, Wouters J, Michiels C and Le Calvé B: Transmembrane (TMEM) protein family members: Poorly characterized even if essential for the metastatic process. Semin Cancer Biol 60: 96-106, 2020.

11. Liu J and Zhu H: TMEM106A inhibits cell proliferation, migration, and induces apoptosis of lung cancer cells. J Cell Biochem: Nov 19, 2018 (Epub ahead of print). doi: 10.1002/jcb.28057.

12. Lang CM, Fellerer K, Schwenk BM, Kuhn PH, Kremmer E, Edbauer D, Capell A and Haass C: Membrane orientation and subcellular localization of transmembrane protein 106B (TMEM106B), a major risk factor for frontotemporal lobar degeneration. J Biol Chem 287: 19355-19365, 2012.

13. Assassi S, Reveille JD, Arnett FC, Weisman MH, Ward MM, Agarwal SK, Gourh P, Bhula J, Sharif R, Sampat K, et al: Whole-blood gene expression profiling in ankylosing spondylitis shows upregulation of toll-like receptor 4 and 5. J Rheumatol 38: 87-98, 2011.

14. Luo X, Han G, Lu R, Guan S, Wang Y, Ju L, Chen L, Shao J and Bian Z: Transmembrane protein 106C promotes the development of hepatocellular carcinoma. J Cell Biochem 121: 4484-4495, 2020.

15. Tang Z, Li C, Kang B, Gao G, Li C and Zhang Z: GEPIA: A web server for cancer and normal gene expression profiling and interactive analyses. Nucleic Acids Res 45: W98-W102, 2017.

16. Therneau TM: Survival Analysis [R package survival version 2.39-5]. 46: 111-112, 2015.
17. R Core Team: A Language And Environment For Statistical Computing. R Foundation for Statistical Computing, Vienna, 2012.

18. Subramanian A, Tamayo P, Mootha VK, Mukherjee S, Ebert BL, Gillette MA, Paulovich A, Pomeroy SL, Golub TR, Lander ES, et al:Gene setenrichmentanalysis: A knowledge-based approach for interpreting genome-wide expression profiles. Proc Natl Acad Sci USA 102: 15545-15550, 2005.

19. Liberzon A, Birger C, Thorvaldsdóttir H, Ghandi M, Mesirov JP and Tamayo P: The Molecular Signatures Database (MSigDB) hallmark gene set collection. Cell Syst 1: 417-425, 2015.

20. Livak KJ and Schmittgen TD: Analysis of relative gene expression data using real-time quantitative PCR and the $2(-\Delta \Delta \mathrm{C}(\mathrm{T}))$ method. Methods 25: 402-408, 2001.

21. Li J, Ma W, Zeng P, Wang J, Geng B, Yang J and Cui Q: LncTar: A tool for predicting the RNA targets of long noncoding RNAs. Brief Bioinform 16: 806-812, 2015.

22. Mo Y, He L, Lai Z, Wan Z, Chen Q, Pan S, Li L, Li D, Huang J, Xue F, et al: LINC01287/miR-298/STAT3 feedback loop regulates growth and the epithelial-to-mesenchymal transition phenotype in hepatocellular carcinoma cells. J Exp Clin Cancer Res 37: 149-149, 2018

23. Zhu S, Huang X, Zhang K, Tan W, Lin Z, He Q, Chen Y and Shang C: Low expression of long noncoding RNA CTC-297N7.9 predicts poor prognosis in patients with hepatocellular carcinoma. Cancer Med 8: 7679-7692, 2019.

24. Duan J, Qian Y, Fu X, Chen M, Liu K, Liu H, Yang J, Liu C and Chang Y: TMEM106C contributes to the malignant characteristics and poor prognosis of hepatocellular carcinoma. Aging (Albany NY) 13: 5585-5606, 2021.

25. Hai T, Wolford CC and Chang YS: ATF3, a hub of the cellular adaptive-response network, in the pathogenesis of diseases: Is modulation of inflammation a unifying component? Gene Expr 15: 1-11, 2010.

26. Yan C and Boyd DD: ATF3 regulates the stability of p53: A link to cancer. Cell Cycle 5: 926-929, 2006.

27. Li J, Yang Z, Chen Z, Bao Y, Zhang H, Fang X and Yang W: ATF3 suppresses ESCC via downregulation of ID1. Oncol Lett 12: 1642-1648, 2016

28. HacklC,Lang SA,Moser C,Mori A,Fichtner-Feigl S, Hellerbrand C, Dietmeier W, Schlitt HJ, Geissler EK and Stoeltzing O: Activating transcription factor-3 (ATF3) functions as a tumor suppressor in colon cancer and is up-regulated upon heat-shock protein 90 (Hsp90) inhibition. BMC Cancer 10: 668, 2010.

29. Lv L, Wei M, Lin P, Chen Z, Gong P, Quan Z and Tang Z: Integrated mRNA and lncRNA expression profiling for exploring metastatic biomarkers of human intrahepatic cholangiocarcinoma. Am J Cancer Res 7: 688-699, 2017.

30. Reed JC: Mechanisms of apoptosis avoidance in cancer. Curr Opin Oncol 11: 68-75, 1999.

31. Shi TY, He J, Wang MY, Zhu ML, Yu KD, Shao ZM, Sun MH, Wu X, Cheng X and Wei Q: CASP7 variants modify susceptibility to cervical cancer in Chinese women. Sci Rep 5: 9225-9225, 2015.

32. Palmerini F, Devilard E, Jarry A, Birg F and Xerri L: Caspase 7 downregulation as an immunohistochemical marker of colonic carcinoma. Hum Pathol 32: 461-467, 2001.

33. Peeney D, Jensen SM, Castro NP, Kumar S, Noonan S, Handler C, Kuznetsov A, Shih J, Tran AD, Salomon DS, et al: TIMP-2 suppresses tumor growth and metastasis in murine model of triple-negative breast cancer. Carcinogenesis 41: 313-325, 2020.

34. Afonja O, Juste D, Das S, Matsuhashi S and Samuels HH: Induction of PDCD4 tumor suppressor gene expression by RAR agonists, antiestrogen and HER-2/neu antagonist in breast cancer cells. Evidence for a role in apoptosis. Oncogene 23: 8135-8145, 2004.

35. Hwang SK, Jeong YJ and Chang Y-C: PDCD4 inhibits lung tumorigenesis by the suppressing p62-Nrf2 signaling pathway and upregulating Keap1 expression. Am J Cancer Res 10: 424-439, 2020 .

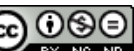

This work is licensed under a Creative Commons Attribution-NonCommercial-NoDerivatives 4.0 International (CC BY-NC-ND 4.0) License. 